Revista

\title{
Multi-Ensayos
}

Vol. 6, núm. 12

ISSN: 2412-3285

https://multiensayos.unan.edu.ni

DOI: https://doi.org/10.5377/multiensayos.v6i12.10117

\section{Rol del docente y estudiante en la educación virtual}

\section{Role of the professor and the student in virtual education}

Marlene Rizo Rodríguez

\section{RESUMEN}

Recibido: 14 de julio de 2020. Aceptado: 10 de agosto de 2020

El avance acelerado de las tecnologías ha ocasionado que en estos tiempos se integren de manera masiva en todos los campos, especialmente en los distintos niveles de la Educación. Este ensayo aborda el rol del docente y estudiante en los entornos virtuales de aprendizaje, cómo influye el rol del docente para el éxito de la actividad formativa centrada en el estudiante y cómo este con su autonomía puede desarrollar habilidades en el uso de las herramientas tecnológicas y manejo de estrategias que le permitan obtener conocimientos y competencias necesarias en el campo profesional en que se está formando, es decir, el propio educando asume el papel protagónico que implica mucha voluntad para el desarrollo de las actividades y trabajos individuales, así como los compartidos. Lo antes mencionado permite aprovechar las fortalezas de todos los participantes en el proceso de colaboración. Además de ello, se comparte experiencia personal relacionada con el rol desempeñado en la facilitación de cursos virtuales en la UNAN Managua - FAREM Estelí.

Palabras clave: entornos virtuales de aprendizaje; rol del docente; rol del estudiante; herramientas tecnológicas.

\begin{abstract}
The recent and accelerating advancement of technology has enhanced the content and quality of all fields in massive ways, especially in the realm of education. This essay addresses the role of the teacher and the student in virtual learning environments, how the role of the teacher influences the success of the student-centered training activity and how, with their autonomy, they can develop skills in the use of technological tools and management of strategies that allow them to obtain the necessary knowledge and skills in the professional field in which they are being trained; that is, the student himself assumes the leading role that implies a lot of will for the development of individual activities and jobs, as well as shared ones. The aforementioned allows to build on the strengths of all participants in the collaboration process. In addition to this, personal experience related to the role played in facilitating virtual courses at UNAN Managua - FAREM Estelí is shared.
\end{abstract}

Keywords: virtual learning environments; role of the teacher; role of the student; technological tools.

1 Docente Facultad Regional Multidisciplinaria, Estelí. UNAN-Managua/FAREM-Estelí. Correo electrónico: mrrodriguez08@ yahoo.es

(c) 2020 Revista Multi-Ensayos. 


\section{INTRODUCCIÓN}

En la actualidad la mayor parte de universidades del mundo ofrecen clases en líneas, cuyo principal eje de apoyo son las Tecnologías de la Información y la Comunicación (TIC), en este sentido la Universidad Nacional Autónoma de Nicaragua, UNAN-Managua está inmersa en esta dirección, apuntando a la calidad educativa y con pertinencia.

El Modelo Educativo, Normativa y Metodología para la Planificación Curricular 2011, aborda la inclusión de las TIC al señalar que " Las Tecnologías de la Información y la Comunicación (TIC), se utilizarán no solo para mejorar las prácticas pedagógicas en el aula, sino para permitirles a los estudiantes otros escenarios de aprendizaje" (UNAN-Managua, 2011, p.33).

En este sentido, la UNAN-Managua promueve el uso y aplicación de las TIC en su quehacer académico. Una de esas formas que estipula el Modelo Educativo dice textualmente: "como medio de enseñanzaaprendizaje, en la planificación de actividades, constituye un recurso fundamental para facilitar a los docentes la construcción del conocimiento en los estudiantes. Así mismo, en el aprendiz, provee un medio de aprendizaje autónomo" (p.33)

Por tanto, en este ensayo se abordan temáticas como ¿Qué es la educación virtual?, ¿Qué elementos intervienen en la educación virtual?, ¿Cuál es el rol del docente en la educación virtual?, ¿Cuál es el rol del estudiante virtual?, así como la Experiencia personal en el rol de docente virtual.

\section{DESARROLLO}

En la sociedad actual del conocimiento se ha evidenciado que el uso de las TIC integradas a la educación han evolucionado vertiginosamente abriendo espacios en distintas modalidades de la enseñanza, siendo estas presenciales, personalizadas, móviles, en la nube, apoyadas con TIC y virtuales. Considerando este último entorno a la enseñanza no presencial que surge cuando el docente utiliza los medios comunicacionales, tecnológicos para generar opciones de interacción de manera sincrónica como asincrónica, es decir, que el mediador y el estudiante interactúan en distintos tiempos, principalmente por el acceso al internet.

En sustentación a los cambios educativos en el siglo XX, Cooperberg, (2005), indica que los medios de la comunicación han revolucionado iniciando desde la época de la correspondencia a la actualidad, donde el aprendizaje es flexible y abierto rodeado de recursos u objetos de aprendizaje que facilitan los mecanismos de mediación.

Es por ello, que en las últimas décadas la educación virtual se ha situado en constante crecimiento y se presenta cada vez más como una necesidad en el contexto de la sociedad donde los rápidos cambios (económicos, sociales, tecnológicos y culturales), el aumento de los conocimientos y las demandas de una educación actualizada se convierten en una exigencia permanente. (Universidad Técnica Nacional, s.f)

Partiendo de lo antes expuesto, conviene definir ¿Qué es la educación virtual? 
La educación virtual es una opción más de actualización permanente que responde a las necesidades de cada persona, ofreciendo así diferentes alternativas o soluciones a una serie de situaciones que la escuela convencional no puede atender. Asimismo, esta modalidad forma parte de los retos que día a día se asumen en el ámbito educativo en la sociedad, sus fundamentos y evolución han permitido que se considere dentro de las ofertas académicas.

Por otro lado, se evidencia la importancia que tiene dicha modalidad en cuanto a la aplicación de las TIC en el proceso de aprendizaje donde los estudiantes tienen mayor acceso, de una manera flexible y colaborativa entre los miembros, por tanto, es importante mencionar los diferentes elementos que actúan en la educación virtual.

Según Camacho (2010) (como se citó en Perdomo \& Perdomo, 2012), refiere que la educación virtual, aunque parezca discordante, sí permite un contacto personal entre el profesor y el estudiante: el intercambio de mensajes escritos y la posibilidad de seguimiento detallado del progreso proporciona al profesor un conocimiento del aprendiz muchas veces mayor que en cursos presenciales; también la información puede adaptarse a los usuarios debido a la modularidad de los contenidos. Por ello, para operar dentro de la educación virtual es indispensable conocer los elementos para impartir el aprendizaje con el uso de la tecnología:

Exploración: el uso de internet o, con más precisión, la World Wide Web como una herramienta de exploración que les abre al profesor y al estudiante las puertas de una fuente inagotable de información y recursos.

Experiencia: el estudiante virtual se ve involucrado en una nueva experiencia social y de aprendizaje que puede incluir comunicaciones directas con su profesor, discusiones con sus compañeros de curso o estudio individual de contenidos a su propio ritmo.

Compromiso: los cursos virtuales ofrecen una oportunidad única al estudiante de compartir experiencias con otros, lo que refuerza el sentido de colaboración y comunidad. Además, el estudiante recibe el control de su tiempo y sus recursos y puede escoger el mejor camino de aprendizaje de acuerdo con sus preferencias y capacidades.

Flexibilidad: desde cualquier lugar y a cualquier hora, los estudiantes pueden tener acceso a sus cursos virtuales.

Actualidad: los profesores tienen la oportunidad de actualizar sus materiales y temas de discusión instantáneamente, lo que hace que los cursos se mantengan frescos y consistentes con la actualidad. Lo anterior supone que el docente, al utilizar las TIC como herramientas en el acto didáctico, enfrente desafíos culturales y técnicos que lleven a un cambio dinámico y complejo en el qué enseñar, cómo enseñar y para qué enseñar. Por ello, la educación virtual apoya a la pedagogía a través de estrategias innovadoras para desarrollar el proceso de aprendizaje al flexibilizar las grandes barreras y bloqueos cognitivos que surgen con la educación tradicional. 
Tomando en cuenta estos elementos, el papel del docente y el estudiante es clave para iniciar y finalizar con éxitos el aprendizaje con la calidad que se requiere, por lo que a continuación se hace una breve reseña de los roles y responsabilidades de cada uno de ellos.

\section{¿Cuál es el papel del docente en la educación virtual?}

De acuerdo Ryan y otros (2000) (citado en Martínez \& Ávila, 2014), el rol central del docente es el de actuar como mediador o intermediario entre los contenidos y la actividad constructivista que despliegan los alumnos para asimilarlos. Los roles y responsabilidades del docente en línea se pueden agrupar en cuatro categorías: pedagógica, social, administrativa y técnica.

- En lo pedagógico: el tutor es un facilitador que contribuye con el conocimiento especializado, focaliza la discusión en puntos críticos, hace las preguntas y responde a las contribuciones de los participantes, le da coherencia a la discusión, sintetiza los puntos destacando los temas emergentes.

- En lo social: necesita habilidades para crear una atmósfera de colaboración que permita generar una comunidad de aprendizaje.

- En el aspecto técnico: debe garantizar que los participantes se sientan cómodos con el software y si es necesario apoyarlos.

- En lo administrativo: conocer el software para poder generar subconferencias, grupos de trabajos y poder mover o borrar mensajes de la conferencia.

Asimismo, citan que también, Cabero (2004), expone clasificaciones a partir de diferentes autores incorporando roles del tipo: organizativo, social e intelectual. En esta misma línea, Adell (1999), establece la siguiente clasificación teniendo en cuenta las nuevas necesidades formativas: Diseñador del currículum: diseño general del curso, planificación de actividades, selección de contenidos y recursos disponibles, etc.

- Proveedor de contenidos: supone la elaboración de materiales de enseñanza en diferentes formatos, caracterizados por la interactividad y la personalización.

- Tutorización: facilitador del aprendizaje.

- Evaluador: tanto de los aprendizajes de los alumnos, como del proceso formativo y de su actuación.

- Técnico: proporcionando soporte de tipo técnico ante las posibles dificultades que los estudiantes se encuentren en el desarrollo del curso (en sus inicios más frecuentemente, y posteriormente durante el progreso en el mismo).

Además, mencionaron que Gisbert (2002) realiza una perspectiva más amplia sobre los roles, funciones y repercusiones, tanto a nivel individual como grupal, que deberán tener en cuenta los docentes de entornos virtuales:

- Consultores de información: Buscadores de materiales y recursos para la información. Soporte a los alumnos para el acceso a la información. Utilizadores experimentados de las herramientas tecnológicas para la búsqueda y recuperación de la información.

- Colaboradores en grupo: Favorecedores de planteamientos y resolución de problemas mediante el trabajo colaborativo, tanto en espacios formales como no formales e informales. Será necesario 
asumir nuevas formas de trabajo colaborativo teniendo en cuenta que nos estamos refiriendo a una colaboración no presencial marcado por las distancias geográficas y por los espacios virtuales.

- Trabajadores solitarios: La tecnología tiene más implicaciones individuales que no grupales, pues las posibilidades de trabajar desde el propio hogar (tele-trabajar) o de formarse desde el propio puesto de trabajo (tele-formación), pueden llevar asociados procesos de soledad y de aislamiento si no se es capaz de aprovechar los espacios virtuales de comunicación y las distintas herramientas de comunicación tanto síncronas como asíncronas (principalmente las primeras).

- Facilitadores del aprendizaje: Las aulas virtuales y los entornos tecnológicos se centran más en el aprendizaje que en la enseñanza entendida en sentido clásico (transmisión de información y de contenidos).

Así, queda claro que los docentes no son transmisores de información, sino facilitadores, proveedores de recursos y buscadores de información.

De igual manera, el docente actúa como un supervisor académico y dirige la vida académica de los estudiantes ajustándose en el perfil de cada estudiante, es decir, debe tomar en cuenta el ritmo de aprendizaje de cada uno de ellos. Por eso, es importante que oriente el proceso de aprendizaje con un buen material educativo e incorpore actividades que fortalezcan la interacción de los participantes.

Asimismo, el docente debe estimular la reflexión, que contemple situaciones de aprendizaje, guiar, proporcionar ayuda e información complementaria: diseñar estrategias para que los alumnos aprendan por sí mismos, corregir sus trabajos y asesorar al estudiante en su proceso de aprendizaje, además evaluar los resultados obtenidos en relación a la materia objeto de estudio.

Es importante, resaltar que el nuevo rol innovador del docente radica principalmente en que guía y motiva al estudiante para que este pueda completar con éxito el proceso de aprendizaje. No obstante, también al final del curso debe realizar un análisis de las experiencias obtenidas a fin de conocer cuáles son los factores facilitadores y obstaculizadores del proceso con el objetivo fortalecer la mejora de la calidad en la educación.

Sin embargo, es necesario que, para que el proceso de aprendizaje virtual sea exitoso, también el estudiante debe estar activo cumpliendo con sus roles, como lo estiman Rugeles C., Mora G., \& Paniagua, (2015, p. 133-137):

En relación con el rol del estudiante en los ambientes educativos mediados por las TIC, Escudero (1992) señala que la definición de los criterios, los valores y los intereses al adoptar las TIC debe obedecer a una planificación que permita desarrollar los procesos pedagógicos, en los que el estudiante virtual desempeña un rol central representado en el conjunto de comportamientos y normas que este debe asumir como actor del proceso educativo.

Algunas características del rol del estudiante virtual que le permiten la generación del conocimiento están directamente relacionadas con la capacidad de autogestión, expresada en la autodisciplina, el autoaprendizaje, el análisis crítico y reflexivo, así como en el trabajo colaborativo, fundamental 
para contribuir al desarrollo del ser en su interacción y aporte con y para otros desde una mirada ética que le permite tomar conciencia de las consecuencias que pueden generar sus acciones.

A continuación, se desarrollan los roles del estudiante que, particularmente, se despliegan en el contexto de la educación virtual dadas las características de las interacciones:

- El rol del estudiante orientado al fortalecimiento de la autodisciplina. Potencia la capacidad para distribuir su tiempo, permite libertad y flexibilidad para el aprovechamiento del aprendizaje mediado por las TIC, conduciendo a la generación de movimientos de los sujetos hacia el logro de sus propias metas. La autodisciplina (Alfie Kohn, 2008) "se puede definir como el control de la propia fuerza de voluntad para cumplir cosas que generalmente se ven como deseables", lo que requiere para la gestión del aprendizaje, una dedicación permanente en las tareas propuestas, fundamentada en la definición de objetivos, su ejecución, seguimiento y control que, acompañados por la decisión, la motivación y la perseverancia, lo conducen hacia el logro de sus metas.

- El rol del estudiante orientado al mejoramiento del auto aprendizaje. Definido según Rugeles, Mora \&Metaute (2013) como la capacidad que desarrolla el individuo para aprender de manera autónoma, activa y participativa, adquiriendo conocimiento y habilidades y fomentando sus propios valores, lo que da como resultado la autoformación del sujeto. Es así como el autoaprendizaje le facilita al estudiante virtual el desarrollo de su capacidad de exigirse a sí mismo, así pues lo involucra en la toma de decisiones como por ejemplo en la distribución de tiempos, la ubicación de espacios, las fuentes de consulta entre las cuales se pueden mencionar bases de datos virtuales especializadas tanto libre como licenciadas, blogs, redes académicas.

De igual forma, el auto aprendizaje favorece los ritmos de estudio y profundización en temas de interés, y lleva al actor a ser autodidacto y auto reflexivo. En el mismo sentido, Gisbert (1999:56) asegura que "el uso de la tecnología nos permite desarrollar nuevas formas de enseñanza y aprendizaje más activas y participativas, a la vez que intuitivas y visuales que favorecen claramente los procesos de auto aprendizaje".

- El rol del estudiante orientado al fortalecimiento del análisis crítico y reflexivo. Definido por Rugeles, Mora, Metaute, (2013) como la habilidad para razonar, analizar y argumentar hechos o acciones que facilitan el desarrollo integral del estudiante y la generación de conocimiento. El análisis crítico y reflexivo busca hacer de la educación un proceso más humanizante desde el punto de vista de la reivindicación de los actores del proceso educativo (estudiante, facilitador) como sujetos pensantes, actuantes, creadores y constructores de saberes individuales y sociales.

Dicha educación humanizadora comprende las dimensiones reflexiva y crítica y, a la vez, ética, donde el pensamiento reflexivo (Escobar, Garcés, 2008) permite la comprensión de lo que aparece en el texto en relación con las experiencias y vivencias del estudiante, en lo relacionado 
al pensamiento crítico; para Martínez, Pascual, (2013) es un proceso cognoscitivo que toma la información, la analiza y está en capacidad de aplicarla en diversos aspectos de la vida.

En el aspecto ético, Franco (2006) lo define como área del conocimiento humano que permite un discernimiento sobre el alcance de las acciones y comportamientos individuales o colectivos relacionados con los aportes constructivos o destructivos para sí, los demás y la naturaleza. Desde el punto de vista de Lipman (1998), estas dimensiones hacen que el facilitador y el estudiante dejen de ser mediadores pasivos entre la teoría y la práctica, para convertirse en mediadores activos que, desde el hacer, reconstruyan críticamente su propia teoría y participen en el desarrollo significativo del conocimiento.

Por lo tanto, el análisis crítico y reflexivo, en el estudiante virtual, deberá ser asumido como una dinámica constante, inmersa en los procesos de enseñanza y aprendizaje, que le permita generar conocimiento aplicado, que en el marco de concepciones éticas de integralidad del ser, se convierta en experiencias significativas.

- El rol del estudiante orientado al mejoramiento del trabajo colaborativo. Pretende romper el aislamiento entre los diferentes actores (Rugeles, Mora, \& Metaute, 2013), permitiendo ver las cualidades individuales que son compartidas entre pares y facilitadores a través de herramientas como el foro, el correo electrónico, las salas de conversación, los Objetos Virtuales de Aprendizaje, (OVA), el chat y el almacenamiento en la nube. En este sentido, Castells (2001) afirma que las personas elaboran sus redes (on line y off line) de acuerdo con sus intereses, valores, afinidades y proyectos, debido a la flexibilidad y al poder de comunicación de Internet, lo cual es fundamental en los procesos de carácter colaborativo en los ambientes educativos mediados por las TIC.

Para el estudiante virtual, el trabajo colaborativo es un factor potenciador del verdadero intercambio de conocimiento, enriquecido con las experiencias del otro, para fortalecer los aprendizajes individuales y el desarrollo de habilidades comunicativas, así como para el desarrollo de destrezas que le permiten ser más recursivo, autónomo y proactivos para la toma de decisiones. El estudiante percibe el trabajo colaborativo como aporte al desarrollo personal, respetando la diferencia y siendo tolerante, pero estableciendo lineamientos definidos con metas comunes.

De igual forma, el trabajo colaborativo le permite exponer su punto de vista a través de posiciones críticas que serán fortalecidas por sus pares y facilitadores, con el fin de buscar intercambio de conocimiento que se pueda poner a disposición de sí mismo y de los demás.

De lo anterior se deduce que el rol del estudiante en los procesos de aprendizaje bajo modalidad virtual con el apoyo de las TIC lo identifica como un sujeto activo, autogestor de sus procesos de enseñanza y aprendizaje con alto compromiso de responsabilidad frente al desarrollo de actividades relacionadas con su formación académica, personal y profesional; con capacidad de optimizar el tiempo y los recursos a su alcance teniendo en cuenta que, en lo relacionado con las TIC, deberá actualizarse permanentemente. 


\section{Experiencia desde el rol docente en el proceso de aprendizaje virtual}

Tomando en cuenta los roles del docente y estudiante virtual mencionados anteriormente por los autores citados, desde mi experiencia personal como facilitadora de la asignatura Informática Básica en la modalidad a distancia virtual, comparto lo siguiente:

Desde el año 2016 la UNAN Managua - FAREM Estelí, inició en la virtualización de algunas asignaturas, entre ellas Informática Básica que se ofrece en el plan de estudios de todas las carreras de pregrado. Desde el año 2017 se me ha dado la oportunidad para facilitar esta asignatura en distintas carreras dirigidas por los departamentos Ciencias de la Educación y Humanidades, Económicas y Administrativas, Tecnológicas y Salud.

Ser docente de la modalidad virtual, aunque parezca fácil, no lo es, se debe tener primero la vocación, el compromiso y la responsabilidad social para guiar el proceso de aprendizaje. Además de todas las funciones que debe cumplir el facilitador en esta modalidad, una de las principales que he puesto en práctica, es generar compromiso por parte de los estudiantes a partir de la claridad del rol que les toca jugar, con el fin de que puedan apropiarse de las competencias necesarias que son la base para el trabajo en el ámbito virtual y, por ende, que sean sujetos activos y constructores de sus propios saberes.

También un elemento importantísimo como parte fundamental que establezco en todo el proceso de aprendizaje virtual es la comunicación fluida, basada en diferentes estrategias como: foros de consultas, mensajes mediante la plataforma, mensajes por WhatsApp, correo electrónico, llamadas telefónicas, con el fin de asistir o conducir las actividades de aprendizaje propuestas en cada una de las semanas, así como para dar respuestas a las consultas y dificultades expuestas por los estudiantes. Esto me ha permitido estar permanentemente disponible para responder lo más inmediato posible con el objetivo de potenciar el proceso de aprendizaje y que el estudiante se estimule mediante la atención, que aunque es virtual, pueda sentir que es presencial.

Es meritorio destacar que la mayoría de los estudiantes también han jugado un rol bastante activo, han sido autodidactas, disciplinados, responsables, éticos, respetuosos, creadores de su propio aprendizaje, lo que les ha permitido finalizar la asignatura de Informática Básica con éxito, con los conocimientos y habilidades necesarias para desempeñarse en el campo académico y laboral. Aunque, hay estudiantes que no logran finalizar con el proceso de aprendizaje, pero ha sido por otros factores que han incidido fuera de los roles que desempeñamos. Sin embargo, se han realizado esfuerzos para poder incorporarlos, en algunos casos con éxito, lamentablemente, otros no se han podido contactar.

Como se menciona antes, al finalizar el curso se evalúa el proceso, en este caso se hace mediante una reflexión partiendo de las siguientes preguntas: ¿Qué aprendí? ¿Cómo aprendí? ¿Qué fortalezas desarrollé? ¿Dónde aplicaré las habilidades adquiridas? ¿Qué debo mejorar?, con esto se consolida el rol activo que han tenido los estudiantes y la satisfacción por parte de mi persona al leer reflexiones positivas $y$, sobre todo, los compromisos que asumen los estudiantes para mejorar las dificultades presentadas en el curso. A continuación, cito algunos de ellos: 
"Debo mejorar la debida administración de mi tiempo, ya que ser estudiante virtual requiere más dedicación para poder aprender", "Creo que tengo que ser constante en el aprendizaje de cada una de estas herramientas ya que la tecnología siempre está en constante cambio, no podemos quedarnos atrás, necesitamos actualizarnos, porque no hay profesión que este divorciada de la tecnología", "En leer más detenidamente la información de lo que dicha plataforma nos pide, en los documentos para realizar una actividad de manera correcta y no tener complicaciones al momento de trabajar", "Aun con los conocimientos que he adquirido hay muchas cosas que todavía me restan por aprender, considero que siempre se puede mejorar, así que dedicaré tiempo para reafirmar mis conocimientos en Word y Excel en particular para poder presentar trabajos de calidad y cumplir con los requerimientos que se me presenten a lo largo de mi carrera y mi vida profesional, en especial me gustaría familiarizarme con las nuevas características que presentan los programas del paquete de Microsoft Office y con sus demás herramientas que presentan un sinnúmero de facilidades para labores específicas".

\section{CONCLUSIÓN}

De acuerdo a lo desarrollado en este ensayo, se puede concluir que las nuevas tecnologías de la información y la comunicación han propiciado una nueva forma de aprendizaje, en donde se generan espacios virtuales que facilitan interacciones entre los participantes de estos procesos educativos. Es importante indicar que el uso y aplicación de aulas virtuales debe ser claro y preciso en el modelo educativo de las instituciones. También es substancial que los docentes y estudiantes logren un cambio de mentalidad en esta forma de aprendizaje virtual.

El ser docente requiere una responsabilidad social, un compromiso, ejercer un rol activo en el ámbito virtual cumpliendo con cada una de las funciones para que los estudiantes adquieran los conocimientos y habilidades en el proceso aprendizaje.

En cuanto al rol del estudiante, este debe ser también un sujeto activo de su propio aprendizaje, debe tomar en cuenta los roles representados en la autodisciplina, auto aprendizaje, en saber analizar, reflexionar y en participar en el trabajo colaborativo, ya esto les permite que su proceso de aprendizaje sea de provecho y calidad.

Por último, se considera que, en los entornos virtuales, los materiales didácticos, las actividades de aprendizajes y los medios de comunicación se hacen importantísimos ya que ellos deben permitir al estudiante analizar, reflexionar e investigar y generar su autoaprendizaje con la calidad que se requiere para enfrentarse al campo laboral.

\section{BIBLIOGRAFÍA}

Cooperberg, A. F. (2005). El proceso de enseñanza-aprendizaje en los entornos de educación a distancia. Obtenido de https://www.um.es/ead/red/3/cooperberg1.pdf

Martínez, L., \& Ávila, Y. (2014). Papel del docente en los entornos virtuales de aprendizaje. Órbita Pedagógica, 2(2 (2016)), 50-52. Obtenido de https://refcale.uleam.edu.ec/index.php/enrevista/ index 
Perdomo, Y., \& Perdomo, N. (2012). Elementos que intervienen en la enseñanza y aprendizaje en línea. Apertura, 66-75. doi:1665-6180

Rugeles Contreras, P. A., Mora González, B., \& Metaute Paniagua, P. M. (2015). El rol del estudiante en los ambientes educativos mediados por las TIC. Lasallista de Investigación, 132-138. doi:17944449

UNAN-Managua. (2011). Modelo Educativo, Normativa y Metodología para la Planificación Curricular 2011. Managua, Nicaragua.

Universidad Técnica Nacional. (s.f). La Educación Virtual en el siglo XXI. Obtenido de http://ftp. campusvirtual.utn.ac.cr/e-learning/La\%20educaci\%C3\%B3n\%20virtual\%20en\%20el\%20siglo\%20 XXI.pdf 